\title{
Physical activity and central adiposity in a cohort of African-American adults
}

\author{
Sean McGrath, Danielle Brazel, Lara Dugas, Guichan Cao, Ramon Durazo-Arvizu and Amy Luke*
}

\begin{abstract}
Background: Visceral adipose tissue (VAT) is known as an independent predictor of cardiometabolic risk and all-cause mortality, while increased physical activity has been shown to improve cardiometabolic risk. The purpose of the present study was to determine whether or not there is an association between objectively-measured physical activity and VAT in a community-based cohort of African-American adults, a population at higher-than-average risk for cardiometabolic diseases.

Methods: Free-living physical activity was quantified using accelerometry while VAT and abdominal subcutaneous fat were estimated using dual $x$-ray absorptiometry in a cohort of African Americans, ages 30-50 years, enrolled in the Modeling the Epidemiologic Transition Study. Univariate and multivariate analyses were used to determine the degree of association comparing moderate-to-vigorous physical activity (MVPA), vigorous activity, and sedentary behavior with measures of central adiposity including VAT, subcutaneous fat, and waist circumference.

Results: A total of 271 individuals with complete data were included in the analyses. Women, on average, had significantly more VAT and abdominal subcutaneous fat than men. There were statistically significant inverse univariate correlations between MVPA and measures of abdominal adiposity $(-0.30, p<0.001)$ and activity counts and adiposity $(-0.31, p<0.001)$ among both sexes. These significant associations remained after controlling for age, sex, and smoking status; neither alcohol consumption nor employment status were associated with abdominal adiposity. Time in sedentary behavior was not meaningfully associated with central adiposity in either sex (women: -0.02 , $p=0.79$; men: $-0.21, p=0.04$ ).

Conclusions: To our knowledge, this study is the first to identify significant inverse associations between MVPA and measures of central adiposity among African American adults from a community-based cohort using an objective measure of physical activity and a validated quantitative imaging technique.
\end{abstract}

Keywords: Visceral adipose tissue, Waist circumference, Moderate-to-vigorous physical activity, Sedentary behavior

\section{Background}

Excess accumulation of visceral adipose tissue (VAT) has been shown to be an independent predictor of insulin resistance, type 2 diabetes, dyslipidemia, and non-alcoholic fatty liver disease [1-9], all of which are associated with increased cardiometabolic risk and are predictors of allcause mortality $[10,11]$. VAT is the most pathogenic fat deposit type and has been found to play a central role in obesity [8]. Recently, it was determined that VAT is a stronger predictor of morbidity and mortality than body weight change [9]. This may be due, at least in part, to the

\footnotetext{
* Correspondence: aluke@luc.edu

Department of Public Health Sciences, Stritch School of Medicine, Loyola University Chicago, 2160 S. First Ave, Maywood, IL 60153, USA
}

metabolic activity of VAT, which increases release of free fatty acids and pro-inflammatory cytokines relative to subcutaneous fat [12-15].

Physical activity has been shown to improve cardiometabolic risk. Laboratory testing shows higher intensity and higher frequency of exercise bouts and training are associated with increased insulin sensitivity, glucose tolerance, and fasting triglycerides [16-19]. Multiple professional and medical organizations provide physical activity guidelines to promote optimal health including the American College of Sports Medicine (ACSM), the American Heart Association (AHA), and the U.S. Department of Health and Human Services. The ACSM and AHA guidelines recommend that U.S. adults partake 
in moderate-intensity aerobic physical activity for at least $30 \mathrm{~min}$ on five or more days per week or vigorousintensity aerobic activity for $20 \mathrm{~min}$ on at least 3 days throughout the week [20]. Federal guidelines are similar, differing only in the recommendation of $75 \mathrm{~min}$ of vigorous aerobic activity over the course of a week [21]. In contrast, sedentary behavior has been associated with increased cardiometabolic risk [22]. In the U.S. it has been repeatedly observed that most adults do not meet the recommendations for physical activity and instead spend a majority of their time either in sedentary or lightintensity activity [23].

Understanding the mechanisms through which physical activity potentially impacts cardiometabolic risk would help inform targeted interventions. Describing the relationship between VAT and physical activity in freeliving populations is a first step in this understanding. To date, much of the research in this area has focused on controlled laboratory experiments or, when conducted at the community- or population-level, has utilized self-reported physical activity and a proxy for VAT and waist circumference. Two recent reports have described inverse associations between objectively measured physical activity and VAT in large samples, though both examined predominantly white participant groups i.e., ADDITION-PRO, a study among Danish individuals at high risk for type 2 diabetes recruited from primary care [24] and the Framingham Third Generation and Omni II cohorts [25]. Little is known, however, about the relationship between physical activity and central adiposity or VAT among African-Americans adults. To gain further insight into the association between physical activity and VAT in this population at high risk of cardiometabolic disease [26], we describe the crosssectional association between physical activity measured using accelerometry and VAT measured using dual $\mathrm{x}$-ray absorptiometry (DXA) in a community-based sample of African-American adults participating in the Modeling the Epidemiologic Transition Study (METS) [27].

\section{Methods}

\section{Study population and institutional review board}

METS is a multi-country, longitudinal cohort study designed to examine the relationship between objectivelymeasured physical activity and weight gain among five community-based samples of adults, predominantly of African descent. Five hundred adults, aged 25 to 45 years, were enrolled between January 2010 and December 2011 within communities in each of the five countries: Ghana, South Africa, Jamaica, Seychelles, and the United States. The study site in the United States is Maywood, IL, a near-western suburb of Chicago in which the majority of residents are African-American. A detailed description of all methods, including recruitment methods in each site, has been previously published [27]. In brief, all city blocks in Maywood were randomized and door-to-door canvassing was conducted until enrollment was complete. Exclusion criteria, as detailed previously, included conditions that would prevent participation in physical activity, such as lower extremity disability and degenerative joint diseases, as well as current pregnancy [27]. The protocol for METS was approved by the Institutional Review Board of Loyola University Chicago and written informed consent was obtained from all participants.

The participants in METS have been followed annually since 2011 for measurement of weight, at a minimum. During the third annual follow-up examination, as a component of the ancillary study, Vitamin D Ancillary, a measurement of body composition using DXA was added to the protocol; anthropometrics and physical activity by accelerometry were measured and change in health status was obtained. An addendum to the original informed consent was signed by all participants covering this additional measurement of body composition. The data presented in this report were all obtained during the third annual follow-up examination for METS.

\section{Measurements}

Physical activity was measured objectively using the Actical accelerometer (Respironics/Mini-Mitter, Bend, OR). Participants were asked to wear the accelerometer behind the right hip on a velcro belt and keep it on 24h per day over the subsequent 8 days with the exceptions of bathing, showering, and swimming. Activity conducted between the hours of 7 am and 11 pm daily were used in these analyses. As reported previously [28], raw data were downloaded from the accelerometers and assessed via a SAS macro program inferring non-wear time from 90 or more minutes of continuous zero activity counts. A valid day of physical activity monitoring was defined as one having $\geq 10 \mathrm{~h}$ of wear time. Only those participant files with four or more valid days of physical activity monitoring were included in these analyses.

Sedentary, moderate, and vigorous activity levels were defined using published criteria: sedentary $<100$ counts per minute (cpm), moderate activity 1535-3959 cpm and vigorous activity $\geq 3960 \mathrm{cpm}[29,30]$. Minutes defined as comprising sedentary, moderate, vigorous, or moderate plus vigorous activity (MVPA) are presented as the total minutes per day accumulated in 1-min intervals. Data are also presented as activity counts per minute as an overall measure of average physical activity intensity. Participants were classified as meeting physical activity guidelines if they accumulated at least $30 \mathrm{~min}$ of MVPA per day on average.

Body composition was measured using DXA (Hologic Discovery W configured with software version 12.1, 
Bedford, MA). Visceral adipose tissue volume and area were calculated using algorithms developed specifically for the Hologic Discovery W DXA instrument [31, 32]. Total abdominal fat in all participants was measured at a site just superior to the iliac crest, as determined from whole body scans, to minimize interference from bone pixels. Traditional DXA estimates of abdominal fat include both subcutaneous and VAT, however, it is possible to differentiate subcutaneous fat in the lateral aspects of the abdomen from muscle or lean tissue [31, 32]. Quantification of lateral subcutaneous fat allowed approximation of anterior and posterior subcutaneous fat, yielding an estimation of total abdominal subcutaneous fat, ie, lateral plus anterior and posterior volumes. Total abdominal subcutaneous fat was then subtracted from total abdominal fat to yield VAT. VAT and subcutaneous fat are expressed as volume $\left(\mathrm{cm}^{3}\right)$. Additionally, VAT area was calculated and used to determine whether or not participants had excess visceral adiposity, defined as $>100 \mathrm{~cm}^{2}$ [33].

Visceral adipose tissue has historically been difficult to measure. Many methods have been used including MRI, computed tomography, ultrasonography, and anthropometric estimations such as waist circumference. These methods have significant drawbacks including cost, radiation exposure, and reproducibility. Dual $\mathrm{x}$-ray absorptiometry measurements of VAT have been shown to be as accurate as computed tomography with less radiation exposure [34] and less expensive than MRI [35].

Weight $(\mathrm{kg})$ and height $(\mathrm{m})$ measurements were made on all participants while wearing light clothing and no shoes. Weight was measured to the nearest $0.1 \mathrm{~kg}$ using a standard calibrated balance (Seca 770, Hamburg, Germany). Height was measured to the nearest $0.1 \mathrm{~cm}$ using a stadiometer (e.g. Invicta Stadiometer, Invicta, London, UK) with the participant's head held in the Frankfort plane. Waist circumference was measured to the nearest $0.1 \mathrm{~cm}$ at the umbilicus and hip at the point of maximum extension of the buttocks. Body mass index (BMI) was calculated as $\mathrm{kg} / \mathrm{m}^{2}$.
A positive smoking status was defined as currently smoking at least one cigarette per day, while positive alcohol consumption was defined as consuming any alcohol during a typical week. Participants were coded as employed if they had worked for pay in the previous month.

\section{Statistical analysis}

All analyses were conducted in Stata Version 12 (College Station, TX, USA). Statistical significance was accepted at $p<0.05$. Descriptive statistics including mean levels and distributions were used to summarize participant characteristics. Prevalences of health status indicators, i.e. obesity and excess visceral adiposity, were calculated for each sex. In addition, descriptive characteristics of the physical activity variables were calculated and included only those individuals with valid data as determined by the inclusion criteria previously described. Univariate analyses were conducted to determine partial correlation coefficients between parameters of physical activity and central adiposity by sex. Multivariate analyses were conducted using linear regression to describe the relationship between physical activity and central adiposity controlling for age, sex, and smoking status in the combined model and for age and smoking status in the sex-specific models.

\section{Results}

\section{Participant characteristics}

A total of 297 participants were examined during the third annual follow-up investigation for METS in Maywood (60\% retention from baseline), of which there were complete DXA and physical activity data on 271 participants available for analysis. There were no differences in baseline weight, height, waist circumference or BMI among those participants who completed the third follow-up examination and those lost to follow-up (Table 1; all $p>0.2$ ). Among women, baseline physical activity parameters, including minutes of MVPA, did not differ between completers of the third examination and

Table 1 Baseline characteristics of participants at the third follow-up examination and those lost to follow-up (mean \pm SD)

\begin{tabular}{|c|c|c|c|c|}
\hline & \multicolumn{2}{|l|}{ Women } & \multicolumn{2}{|l|}{ Men } \\
\hline & $\begin{array}{l}\text { Third Follow-up Examination } \\
(n=161)\end{array}$ & $\begin{array}{l}\text { Lost to } \\
\text { Follow-up } \\
(n=96)\end{array}$ & $\begin{array}{l}\text { Third Follow-up Examination } \\
(n=110)\end{array}$ & $\begin{array}{l}\text { Lost to } \\
\text { Follow-up } \\
(n=135)\end{array}$ \\
\hline Age (y) & $35.0 \pm 6.3$ & $35.0 \pm 6.2$ & $36.7 \pm 6.3^{*}$ & $34.7 \pm 6.1^{*}$ \\
\hline Weight (kg) & $92.1 \pm 25.5$ & $90.9 \pm 22.8$ & $92.8 \pm 27.4$ & $92.8 \pm 22.5$ \\
\hline Height (cm) & $163.8 \pm 6.3$ & $164.3 \pm 6.0$ & $175.9 \pm 6.5$ & $177.2 \pm 6.6$ \\
\hline Body Mass Index (kg/m²) & $34.3 \pm 9.2$ & $33.7 \pm 8.3$ & $29.9 \pm 8.2$ & $29.6 \pm 6.9$ \\
\hline Waist Circumference $(\mathrm{cm})$ & $102.6 \pm 20.7$ & $100.5 \pm 17.8$ & $98.4 \pm 22.9$ & $96.2 \pm 20.2$ \\
\hline MVPA (min/d) & $14.5 \pm 17.2$ & $16.6 \pm 18.9$ & $28.8 \pm 27.0$ & $37.6 \pm 41.3$ \\
\hline Vigorous PA (min/d) & $1.4 \pm 3.4$ & $1.2 \pm 3.1$ & $2.7 \pm 4.6^{*}$ & $6.3 \pm 14.1^{*}$ \\
\hline
\end{tabular}

${ }^{*} p<0.05$ 
those lost to follow-up. Among men, however, baseline age was statistically significantly older (36.7 \pm 6.3 vs $34.7 \pm 6.1$ $\mathrm{y}$, respectively; $p<0.05$ ) and moderate-to-vigorous physical activity was lower among those who remained in METS compared to those lost to follow-up (38.0 \pm 3.9 vs $28.5 \pm 2.5 \mathrm{~min} /$ day; $\mathrm{p}<0.05)$. These differences could not be explained by the employment status or employment type of the completers versus those lost to follow-up.

Characteristics of those participants who completed the third annual follow-up examination, for whom there were complete body composition and physical activity data, are presented in Table 2. The study population is composed of middle-aged African Americans with an average BMI of $35.0 \mathrm{~kg} / \mathrm{m}^{2}$ for women and $30.2 \mathrm{~kg} / \mathrm{m}^{2}$ for men. The cohort had very high prevalences of whole-body obesity, as defined by BMI $\geq 30 \mathrm{~kg} / \mathrm{m}^{2}$, and of excess visceral adiposity, especially among the women. The participants reported relatively high proportions of current smokers and consumers of alcohol, particularly among the men. A large majority of both women and men were employed at the time of the third follow-up examination.

\section{Body composition by DXA}

As presented in Table 2, participants had an average of $634 \mathrm{~cm}^{3}$ of visceral adipose tissue and $2007 \mathrm{~cm}^{3}$ of abdominal subcutaneous adipose tissue. Men had less subcutaneous adipose and visceral adipose tissue than women, with men having $1323 \mathrm{~cm}^{3}$ of SAT and $562 \mathrm{~cm}^{3}$ of VAT compared to women who had $2504 \mathrm{~cm}^{3}$ of SAT and $686 \mathrm{~cm}^{3}$ of VAT.

\section{Physical activity by accelerometer}

On average, participants undertook 19.6 min per day of MVPA, $1.8 \mathrm{~min}$ of vigorous intensity activity, and $213.3 \mathrm{~min}$ of sedentary behavior. Men tended to participate in twice the amount of MVPA and vigorous intensity activity as women. On average men partook in 29.8 min per day of MVPA and 2.9 min per day of vigorous activity as compared to women who averaged $13.0 \mathrm{~min}$ per day of MVPA and $1.0 \mathrm{~min}$ per day of vigorous activity. Among men, $36.1 \%$ met the U.S. physical activity guidelines of $30 \mathrm{~min} /$ day of MVPA while only $15.3 \%$ of the women met the same guidelines $(p<0.001)$.

Table 2 Participant characteristics at third follow-up examination (mean \pm SD; \%)

\begin{tabular}{|c|c|c|c|}
\hline & $\begin{array}{l}\text { Women } \\
(n=161)\end{array}$ & $\begin{array}{l}\text { Men } \\
(n=110)\end{array}$ & $\begin{array}{l}\text { Total } \\
(n=271)\end{array}$ \\
\hline Age (y) & $38.1 \pm 6.4$ & $39.7 \pm 6.6$ & $38.8 \pm 6.5$ \\
\hline Weight (kg) & $91.0 \pm 24.1$ & $89.6 \pm 26.1$ & $90.4 \pm 24.9$ \\
\hline Body mass index $\left(\mathrm{kg} / \mathrm{m}^{2}\right)$ & $35.0 \pm 9.2$ & $30.2 \pm 8.3$ & $33.0 \pm 9.1$ \\
\hline Waist circumference (cm) & $104.8 \pm 19.5$ & $100.1 \pm 20.8$ & $102.9 \pm 20.1$ \\
\hline Hip circumference $(\mathrm{cm})$ & $119.7 \pm 16.5$ & $110.5 \pm 15.0$ & $115.9 \pm 16.5$ \\
\hline Whole body lean mass (kg) & $55.0 \pm 10.4$ & $67.8 \pm 12.1$ & $60.2 \pm 12.8$ \\
\hline Whole body fat mass(kg) & $36.0 \pm 15.3$ & $21.8 \pm 15.7$ & $30.2 \pm 16.9$ \\
\hline Body fat (\%) & $38.4 \pm 7.9$ & $22.2 \pm 9.5$ & $31.8 \pm 11.7$ \\
\hline Visceral adipose tissue $\left(\mathrm{cm}^{3}\right)$ & $686 \pm 366$ & $562 \pm 362$ & $636 \pm 369$ \\
\hline Abdominal subcutaneous tissue $\left(\mathrm{cm}^{3}\right)$ & $2504 \pm 902$ & $1323 \pm 1039$ & $2025 \pm 1120$ \\
\hline$\%$ Obese & 68.8 & 40.3 & 57.3 \\
\hline \% Excess Visceral Adiposity ${ }^{a}$ & 64.8 & 45.4 & 57.0 \\
\hline Activity counts (ct/min) & $128.2 \pm 69.2$ & $185.0 \pm 115.1$ & $151.3 \pm 94.7$ \\
\hline Moderate-to-vigorous intensity activity $(\mathrm{min} / \mathrm{d})^{\mathrm{b}}$ & $13.0 \pm 13.6$ & $29.8 \pm 30.2$ & $19.8 \pm 23.3$ \\
\hline Vigorous intensity activity $(\mathrm{min} / \mathrm{d})^{\mathrm{b}}$ & $1.0 \pm 2.5$ & $2.9 \pm 5.9$ & $1.8 \pm 4.3$ \\
\hline Sedentary behavior $(\mathrm{min} / \mathrm{d})^{\mathrm{b}}$ & $214.4 \pm 46.0$ & $209.0 \pm 52.7$ & $212 \pm 49$ \\
\hline$\%$ Meeting PA Guidelines ${ }^{c}$ & 15.3 & 36.1 & 23.7 \\
\hline$\%$ Current Smoker ${ }^{d}$ & 27.5 & 60.9 & 41.1 \\
\hline$\%$ Alcohol Consumption $^{\mathrm{e}}$ & 36.9 & 61.8 & 47.0 \\
\hline$\%$ Employed $^{f}$ & 81.3 & 82.6 & 81.8 \\
\hline
\end{tabular}

${ }^{a}$ Defined as $>100 \mathrm{~cm}^{2}$ VAT

${ }^{\mathrm{b}}$ As measured in 1-min bouts

'Defined as $>30 \mathrm{~min} / \mathrm{d}$ of MVPA

${ }^{\mathrm{d}}$ Defined as currently smoking at least 1 cigarette per day

eDefined as consuming any alcoholic beverages during a typical week

fDefined as working for pay in the previous month 
Relationship between visceral adipose tissue and physical activity

Table 3 presents the univariate correlation coefficients between measures of physical activity and measures of central adiposity. There were statistically significant negative associations between MVPA and VAT, abdominal subcutaneous adipose tissue, and waist circumference in both sexes. Likewise, activity counts were consistently significantly associated with measures of central adiposity in both women and men. In contrast, correlations between vigorous intensity activity or sedentary behavior and central adiposity differed between the sexes. Among women, vigorous activity was significantly, inversely associated with the measures of adiposity while sedentary behavior was not significantly associated at all. In men, no statistically significant association was found between vigorous activity and central adiposity while sedentary behavior was found to be inversely associated. It must be noted, however, that the association between sedentary behavior and adiposity was opposite from what would be expected, i.e., an inverse association was observed rather than the anticipated positive association.

Results of the multivariate regression analyses are presented in Table 4, for the total cohort as well as for women and men, separately. In the total cohort, after controlling for age, sex, and smoking status, statistically significant inverse associations were observed between all measures of physical activity and the outcome variables of VAT, SAT and waist circumference (all $p<0.01)$. When stratified by sex and after controlling for age and smoking status, slight differences in the associations between physical activity parameters and measures of central adiposity were observed between the women and the men. Among women, statistically significant associations were observed for all measures of activity and of adiposity; smoking status was not independently associated with VAT, SAT or waist circumference. Among men, however, the associations between activity counts and MVPA and measures of central adiposity remained statistically significant, whereas vigorous activity was only marginally associated. In contrast to women, smoking status was independently associated with VAT and SAT among men. Only a relatively small proportion of the variance in VAT, SAT or waist circumference could be explained by the covariates used in the sex-stratified models. Over $30 \%$ of the variance in SAT could be explained by the model which included both sexes, however, this is due to the significant difference in SAT volume between women and men.

No statistically significant association was observed between sedentary behavior and any measure of central adiposity. Likewise, alcohol consumption and employment status were not included in the multivariate analyses due to a consistent lack of association with outcomes $(p>0.5)$.

\section{Discussion}

These analyses demonstrate significant inverse associations between physical activity parameters and all measures of central adiposity in this community-based sample of African-American adults, more than half of whom were classified as obese based on BMI. The

Table 3 Univariate associations between markers of central adiposity and physical activity parameters by sex (correlation coefficient [p-value])

\begin{tabular}{|c|c|c|c|}
\hline & $\begin{array}{l}\text { VAT } \\
\left(\mathrm{cm}^{3}\right)\end{array}$ & $\begin{array}{l}\text { SAT } \\
\left(\mathrm{cm}^{3}\right)\end{array}$ & Waist circumference $(\mathrm{cm})$ \\
\hline \multicolumn{4}{|l|}{ Total $(n=271)$} \\
\hline Activity counts (ct/min) & $-0.31[0.001]$ & $-0.34[0.001]$ & $-0.23[0.001]$ \\
\hline $\operatorname{MVPA}(\mathrm{min} / \mathrm{d})^{\mathrm{a}}$ & $-0.30[0.001]$ & $-0.37[0.001]$ & $-0.24[0.001]$ \\
\hline Vigorous intensity activity $(\mathrm{min} / \mathrm{d})^{\mathrm{a}}$ & $-0.21[0.001]$ & $-0.25[0.001]$ & $-0.19[0.002]$ \\
\hline Sedentary behavior $(\mathrm{min} / \mathrm{d})^{a}$ & $-0.09[0.13]$ & $-0.09[0.13]$ & $-0.07[0.25]$ \\
\hline \multicolumn{4}{|l|}{ Women $(n=161)$} \\
\hline Activity counts (ct/min) & $-0.24[0.002]$ & $-0.16[0.04]$ & $-0.15[0.05]$ \\
\hline $\operatorname{MVPA}(\min / \mathrm{d})^{\mathrm{a}}$ & $-0.25[0.001]$ & $-0.21[0.008]$ & $-0.19[0.02]$ \\
\hline Vigorous intensity activity $(\mathrm{min} / \mathrm{d})^{\mathrm{a}}$ & $-0.24[0.003]$ & $-0.21[0.008]$ & $-0.20[0.01]$ \\
\hline Sedentary behavior $(\mathrm{min} / \mathrm{d})^{\mathrm{a}}$ & $-0.02[0.79]$ & $-0.06[0.49]$ & $-0.03[0.71]$ \\
\hline \multicolumn{4}{|l|}{ Men $(n=110)$} \\
\hline Activity counts (ct/min) & $-0.32[0.001]$ & $-0.28[0.003]$ & $-0.27[0.005]$ \\
\hline $\operatorname{MVPA}(\mathrm{min} / \mathrm{d})^{\mathrm{a}}$ & $-0.29[0.002]$ & $-0.26[0.006]$ & $-0.24[0.01]$ \\
\hline Vigorous intensity activity $(\mathrm{min} / \mathrm{d})^{a}$ & $-0.15[0.11]$ & $-0.16[0.09]$ & $-0.18[0.06]$ \\
\hline Sedentary behavior $(\mathrm{min} / \mathrm{d})^{\mathrm{a}}$ & $-0.21[0.03]$ & $-0.20[0.03]$ & $-0.19[0.04]$ \\
\hline
\end{tabular}

${ }^{\mathrm{a}}$ As measured in 1-min bouts 
Table 4 Regression coefficients and intercepts for the relationship between parameters of physical activity and central adiposity, adjusting for age and smoking status, total cohort \& by sex ( $p$-value)

\begin{tabular}{|c|c|c|c|c|c|c|c|}
\hline & Intercept & $\begin{array}{l}\beta_{1} \\
\text { Activity Parameter }\end{array}$ & $\begin{array}{l}\beta_{2} \\
\text { Age }\end{array}$ & $\begin{array}{l}\beta_{3} \\
\text { Sex }\end{array}$ & $\begin{array}{l}\beta_{4} \\
\text { Smoking Status }\end{array}$ & SEE & $R^{2}$ \\
\hline \multicolumn{8}{|l|}{ Total $(n=271)$} \\
\hline \multicolumn{8}{|l|}{$\operatorname{VAT}\left(\mathrm{cm}^{3}\right)$} \\
\hline Activity Counts (ct/min) & $\begin{array}{l}484 \\
(0.001)\end{array}$ & $\begin{array}{l}-1.1 \\
(0.001)\end{array}$ & $\begin{array}{l}7.9 \\
(0.02)\end{array}$ & $\begin{array}{l}56.0 \\
(0.25)\end{array}$ & $\begin{array}{l}-69.7 \\
(0.14)\end{array}$ & 348 & 0.13 \\
\hline MVPA (min/d) & $\begin{array}{l}434 \\
(0.003)\end{array}$ & $\begin{array}{l}-4.0 \\
(0.001)\end{array}$ & $\begin{array}{l}7.1 \\
(0.03)\end{array}$ & $\begin{array}{l}49.4 \\
(0.32)\end{array}$ & $\begin{array}{l}-65.2 \\
(0.17)\end{array}$ & 351 & 0.11 \\
\hline Vigorous PA (min/d) & $\begin{array}{l}311 \\
(0.03)\end{array}$ & $\begin{array}{l}-15.5 \\
(0.003)\end{array}$ & $\begin{array}{l}8.7 \\
(0.01)\end{array}$ & $\begin{array}{l}83.4 \\
(0.09)\end{array}$ & $\begin{array}{l}-84.4 \\
(0.08)\end{array}$ & 355 & 0.09 \\
\hline \multicolumn{8}{|l|}{ SAT $\left(\mathrm{cm}^{3}\right)$} \\
\hline Activity Counts (ct/min) & $\begin{array}{l}1701 \\
(0.001)\end{array}$ & $\begin{array}{l}-2.2 \\
(0.001)\end{array}$ & $\begin{array}{l}5.8 \\
(0.51)\end{array}$ & $\begin{array}{l}956 \\
(0.001)\end{array}$ & $\begin{array}{l}-325 \\
(0.009)\end{array}$ & 932 & 0.32 \\
\hline MVPA (min/d) & $\begin{array}{l}1630 \\
(0.001)\end{array}$ & $\begin{array}{l}-9.1 \\
(0.001)\end{array}$ & $\begin{array}{l}3.9 \\
(0.66)\end{array}$ & $\begin{array}{l}928 \\
(0.001)\end{array}$ & $\begin{array}{l}-311 \\
(0.01)\end{array}$ & 932 & 0.32 \\
\hline Vigorous PA (min/d) & $\begin{array}{l}1351 \\
(0.001)\end{array}$ & $\begin{array}{l}-37.6 \\
(0.007)\end{array}$ & $\begin{array}{l}7.5 \\
(0.39)\end{array}$ & $\begin{array}{l}1003 \\
(0.001)\end{array}$ & $\begin{array}{l}-355 \\
(0.005)\end{array}$ & 940 & 0.31 \\
\hline \multicolumn{8}{|l|}{ Waist Circumference (cm) } \\
\hline Activity Counts (ct/min) & $\begin{array}{l}98.8 \\
(0.001)\end{array}$ & $\begin{array}{l}-0.05 \\
(0.001)\end{array}$ & $\begin{array}{l}0.28 \\
(0.13)\end{array}$ & $\begin{array}{l}1.95 \\
(0.47)\end{array}$ & $\begin{array}{l}-2.89 \\
(0.27)\end{array}$ & 19.6 & 0.07 \\
\hline MVPA (min/d) & $\begin{array}{l}97.1 \\
(0.001)\end{array}$ & $\begin{array}{l}-0.18 \\
(0.001)\end{array}$ & $\begin{array}{l}0.24 \\
(0.19)\end{array}$ & $\begin{array}{l}1.51 \\
(0.58)\end{array}$ & $\begin{array}{l}-2.63 \\
(0.31)\end{array}$ & 19.7 & 0.07 \\
\hline Vigorous PA (min/d) & $\begin{array}{l}91.8 \\
(0.001)\end{array}$ & $\begin{array}{l}-0.83 \\
(0.004)\end{array}$ & $\begin{array}{l}0.32 \\
(0.09)\end{array}$ & $\begin{array}{l}2.81 \\
(0.29)\end{array}$ & $\begin{array}{l}-3.48 \\
(0.18)\end{array}$ & 19.7 & 0.6 \\
\hline \multicolumn{8}{|l|}{ Women $(n=161)$} \\
\hline \multicolumn{8}{|l|}{ VAT $\left(\mathrm{cm}^{3}\right)$} \\
\hline Activity Counts (ct/min) & $\begin{array}{l}506 \\
(0.004)\end{array}$ & $\begin{array}{l}-1.4 \\
(0.001)\end{array}$ & $\begin{array}{l}9.6 \\
(0.03)\end{array}$ & - & $\begin{array}{l}-6.6 \\
(0.92)\end{array}$ & 355 & 0.09 \\
\hline MVPA (min/d) & $\begin{array}{l}429 \\
(0.01)\end{array}$ & $\begin{array}{l}-7.8 \\
(0.001)\end{array}$ & $\begin{array}{l}9.4 \\
(0.03)\end{array}$ & - & $\begin{array}{l}0.23 \\
(0.99)\end{array}$ & 354 & 0.09 \\
\hline Vigorous PA (min/d) & $\begin{array}{l}431 \\
(0.01)\end{array}$ & $\begin{array}{l}-39.6 \\
(0.001)\end{array}$ & $\begin{array}{l}7.9 \\
(0.07)\end{array}$ & - & $\begin{array}{l}-17.0 \\
(0.79)\end{array}$ & 356 & 0.08 \\
\hline \multicolumn{8}{|l|}{ SAT $\left(\mathrm{cm}^{3}\right)$} \\
\hline Activity Counts (ct/min) & $\begin{array}{l}2645 \\
(0.001)\end{array}$ & $\begin{array}{l}-2.0 \\
(0.07)\end{array}$ & $\begin{array}{l}4.8 \\
(0.67)\end{array}$ & - & $\begin{array}{l}-257 \\
(0.12)\end{array}$ & 898 & 0.04 \\
\hline MVPA (min/d) & $\begin{array}{l}2551 \\
(0.001)\end{array}$ & $\begin{array}{l}-13.4 \\
(0.02)\end{array}$ & $\begin{array}{l}5.0 \\
(0.65)\end{array}$ & - & $\begin{array}{l}-241 \\
(0.14)\end{array}$ & 890 & 0.05 \\
\hline Vigorous PA (min/d) & $\begin{array}{l}2566 \\
(0.001)\end{array}$ & $\begin{array}{l}-79.8 \\
(0.009)\end{array}$ & $\begin{array}{l}2.5 \\
(0.82)\end{array}$ & - & $\begin{array}{l}-270 \\
(0.09)\end{array}$ & 888 & 0.06 \\
\hline \multicolumn{8}{|l|}{ Waist Circumference (cm) } \\
\hline Activity Counts (ct/min) & $\begin{array}{l}99.8 \\
(0.001)\end{array}$ & $\begin{array}{l}-0.05 \\
(0.04)\end{array}$ & $\begin{array}{l}0.29 \\
(0.23)\end{array}$ & - & $\begin{array}{l}0.35 \\
(0.92)\end{array}$ & 19.5 & 0.03 \\
\hline MVPA (min/d) & $\begin{array}{l}97.3 \\
(0.001)\end{array}$ & $\begin{array}{l}-0.30 \\
(0.01)\end{array}$ & $\begin{array}{l}0.29 \\
(0.22)\end{array}$ & - & $\begin{array}{l}0.67 \\
(0.84)\end{array}$ & 19.4 & 0.04 \\
\hline Vigorous PA (min/d) & $\begin{array}{l}97.5 \\
(0.001)\end{array}$ & $\begin{array}{l}-1.64 \\
(0.01)\end{array}$ & $\begin{array}{l}0.23 \\
(0.33)\end{array}$ & - & $\begin{array}{l}0.03 \\
(0.99)\end{array}$ & 19.4 & 0.04 \\
\hline \multicolumn{8}{|l|}{ Men $(n=110)$} \\
\hline \multicolumn{8}{|l|}{$\operatorname{VAT}\left(\mathrm{cm}^{3}\right)$} \\
\hline Activity Counts (ct/min) & $\begin{array}{l}535 \\
(0.01)\end{array}$ & $\begin{array}{l}-0.88 \\
(0.003)\end{array}$ & $\begin{array}{l}7.0 \\
(0.17)\end{array}$ & - & $\begin{array}{l}-146 \\
(0.03)\end{array}$ & 339 & 0.15 \\
\hline MVPA (min/d) & $\begin{array}{l}478 \\
(0.03)\end{array}$ & $\begin{array}{l}-2.9 \\
(0.01)\end{array}$ & $\begin{array}{l}6.4 \\
(0.22)\end{array}$ & - & $\begin{array}{l}-141 \\
(0.04)\end{array}$ & 343 & 0.13 \\
\hline
\end{tabular}


Table 4 Regression coefficients and intercepts for the relationship between parameters of physical activity and central adiposity, adjusting for age and smoking status, total cohort \& by sex ( $p$-value) (Continued)

\begin{tabular}{|c|c|c|c|c|c|c|c|}
\hline & Intercept & $\begin{array}{l}\beta_{1} \\
\text { Activity Parameter }\end{array}$ & $\begin{array}{l}\beta_{2} \\
\text { Age }\end{array}$ & $\begin{array}{l}\beta_{3} \\
\text { Sex }\end{array}$ & $\begin{array}{l}\beta_{4} \\
\text { Smoking Status }\end{array}$ & SEE & $R^{2}$ \\
\hline Vigorous PA (min/d) & $\begin{array}{l}295 \\
(0.15)\end{array}$ & $\begin{array}{l}-10.0 \\
(0.08)\end{array}$ & $\begin{array}{l}10.0 \\
(0.05)\end{array}$ & - & $\begin{array}{l}-168 \\
(0.02)\end{array}$ & 349 & 0.10 \\
\hline \multicolumn{8}{|l|}{$\operatorname{SAT}\left(\mathrm{cm}^{3}\right)$} \\
\hline Activity Counts (ct/min) & $\begin{array}{l}1717 \\
(0.01)\end{array}$ & $\begin{array}{l}-2.3 \\
(0.01)\end{array}$ & $\begin{array}{l}7.1 \\
(0.63)\end{array}$ & - & $\begin{array}{l}-410 \\
(0.04)\end{array}$ & 996 & 0.11 \\
\hline MVPA (min/d) & $\begin{array}{l}1582 \\
(0.01)\end{array}$ & $\begin{array}{l}-7.8 \\
(0.02)\end{array}$ & $\begin{array}{l}5.4 \\
(0.72)\end{array}$ & - & $\begin{array}{l}-395 \\
(0.05)\end{array}$ & 1004 & 0.10 \\
\hline Vigorous PA (min/d) & $\begin{array}{l}1096 \\
(0.07)\end{array}$ & $\begin{array}{l}-28.3 \\
(0.09)\end{array}$ & $\begin{array}{l}15.0 \\
(0.31)\end{array}$ & - & $\begin{array}{l}-467 \\
(0.02)\end{array}$ & 1016 & 0.08 \\
\hline \multicolumn{8}{|l|}{ Waist Circumference (cm) } \\
\hline Activity Counts (ct/min) & $\begin{array}{l}99.1 \\
(0.001)\end{array}$ & $\begin{array}{l}-0.04 \\
(0.02)\end{array}$ & $\begin{array}{l}0.32 \\
(0.28)\end{array}$ & - & $\begin{array}{l}-6.8 \\
(0.09)\end{array}$ & 20.0 & 0.10 \\
\hline MVPA (min/d) & $\begin{array}{l}96.4 \\
(0.001)\end{array}$ & $\begin{array}{l}-0.14 \\
(0.04)\end{array}$ & $\begin{array}{l}0.29 \\
(0.34)\end{array}$ & - & $\begin{array}{l}-6.6 \\
(0.10)\end{array}$ & 20.1 & 0.09 \\
\hline Vigorous PA (min/d) & $\begin{array}{l}87.9 \\
(0.001)\end{array}$ & $\begin{array}{l}-0.64 \\
(0.05)\end{array}$ & $\begin{array}{l}0.47 \\
(0.11)\end{array}$ & - & $\begin{array}{l}-7.9 \\
(0.05)\end{array}$ & 20.2 & 0.08 \\
\hline
\end{tabular}

strongest of these univariate associations in both women and men were between MVPA and VAT, while abdominal SAT and waist circumference, indirect measures of central adiposity, were also significantly negatively correlated with MVPA. These univariate associations remained statistically significant after controlling for age, sex, and current smoking status. In contrast to expectations, however, time spent in sedentary behavior was not positively associated with any parameter of central adiposity.

Similar to the current study, Murabito et al. [25] examined the relationship between objectively measured physical activity and VAT among men and women enrolled in the Framingham Third Generation and Omni II cohorts. In concert with our results, these authors observed an inverse association between MVPA and VAT for both men and women with no significant relationship identified for sedentary time and VAT.

Results from other studies have produced mixed results regarding an association between abdominal adiposity and objective measures of physical activity. One study conducted by Smith et al. [36] using computed tomography to quantify VAT and concluded that physical activity, when measured using accelerometry and expressed as MVPA or total active time, was not associated with VAT among men but was among women. In contrast, Philipsen et al. [24] conducted a similar study measuring VAT using ultrasonography and, similar to the present study's results, reported an inverse association between physical activity and visceral adipose tissue in both sexes. Interestingly, both the Smith et al. and Philipsen et al. studies combined physical activity from all intensity categories into one global physical activity classification. The present analyses were conducted with the physical activity intensity categories kept separate in order to assess whether or not MVPA or vigorous physical activity versus minutes of sedentary behavior impacted central adiposity. Our findings have shown that statistically significant associations exist for MVPA and all parameters of central adiposity, which in this case include VAT, subcutaneous adipose tissue, and waist circumference. The present study found no significant relationship between vigorous activity and adiposity among men. Among women, however, there were significant associations between minutes of vigorous activity and measures of adiposity, despite the fact that women only recorded $1 \mathrm{~min}$ of vigorous activity per day on average.

Previously, well-controlled studies have investigated the impact of initiating exercise programs on the reduction of VAT $[37,38]$ and found a dose-response relationship between physical activity intensity and the reduction in VAT. Individuals who exercise at higher intensities have greater reductions in VAT than those exercising moderately or following dietary restrictions only $[37,38]$. One study concluded the greatest reduction in mortality risks when comparing inactive to moderately active groups across all levels of adiposity [39]. Moving from the inactive to the moderately inactive group required a physical activity energy expenditure between 90 and $110 \mathrm{kcal} /$ day, or in other words, about 20 min of brisk walking per day. This indicates that even small increases in activity in otherwise inactive individuals may significantly benefit health on a broad scale. Interestingly, another study showed the combination of aerobic exercise and strength training has a modest reduction of VAT but both separately had a significant VAT decrease [8]. This study found a greater 
effect in men, possibly due to men having more abdominal fat at baseline and thus making them more susceptible to abdominal adiposity changes. This same study also showed that exercise programs without caloric restriction can reduce VAT. Another recent investigation in overweight and obese individuals found that diet restriction resulted in greater weight loss but exercise produced greater VAT reduction [9]. The authors explain this is likely due to caloric restriction decreasing both muscle and fat mass versus exercise increases muscle mass while decreasing fat mass. This suggests that VAT may be a more useful clinical marker of obesity management and intervention. In our study, the lack of association between vigorous physical activity and VAT among men may reflect the overall low levels of objectively measured vigorous activity we captured in this study. It should, however, be noted that the present study was designed to capture habitual physical activity levels rather than exercise intensity or intervention, and these data indicate low exercise levels.

Our study had several limitations; the first being the cross-sectional nature. It is not possible to infer whether increasing physical activity might decrease VAT. Understanding changes in abdominal adiposity over time in relationship to habitual physical activity levels will provide guidance for clinical applications. Secondly, although statistically significant associations were seen, our participants participated in very little vigorous activity; only $1.8 \mathrm{~min}$ per day on average for men and women combined. Having a population that engaged in more vigorous activity may allow for stronger associations. The present population-based study was designed as an observational assessment rather than any type of exercise intervention, therefore, caution should be taken when interpreting these results through the lens of exercise.

Strengths of the present study include the objective measurement of both physical activity and abdominal fat and the community-based sample of study participants. The African-American participants enrolled in the present study mirror populations at increased risk for cardiometabolic diseases including type 2 diabetes. The negative health impact of increased levels of VAT is substantial. Increased volume of VAT has been shown to increase dyslipidemia, insulin resistance, non-alcoholic fatty liver disease and all-cause mortality $[1-3,6,8,10]$, therefore, it is important to identify the level of MVPA and vigorous intensity physical activity required to reduce VAT in this population. This study provides a baseline for further prospective analysis to determine the association between physical activity and visceral adipose tissue and its effect on metabolic risk. Longitudinal analyses are needed to fully understand how visceral adipose tissue changes with physical activity.

\section{Conclusions}

To our knowledge, this study is the first to identify associations between parameters of physical activity and measures of central adiposity among African American adults from a community-based cohort. Major strengths of this study include objective measure of physical activity and a validated quantitative imaging technique. This study is unique in that normal physical activity level, rather an exercise intervention, was assessed and may have important clinical implications for future obesity management.

\section{Abbreviations}

ACSM: American College of Sports Medicine; AHA: American Heart Association; DXA: Dual x-ray absorptiometry; METS: Modeling the epidemiologic transition study; MRI: Magnetic resonance imaging; MVPA: Moderate-to-vigorous physical activity; SAS: Statistical analysis system; SAT: Subcutaneous adipose tissue; VAT: Visceral adipose tissue; WDXA: W configured dual x-ray absorptiometry

\section{Acknowledgements}

The authors would like to acknowledge the clinic staff members as well as the study participants.

Funding

This research was supported in part by funding from the National Institutes of Health (R01DK080763 and R01DK090360).

\section{Availability of data and materials}

The data sets analyzed during this study are available from the corresponding author on reasonable request.

\section{Authors' contributions}

$\mathrm{AL}, \mathrm{SM}, \mathrm{LD}, \mathrm{RDA}$ all conceived the idea and contributed equally to the manuscript. $S M, A L$, and GC performed the analysis, tables, and figures. All authors (AL, SM, LD, $\mathrm{GC}, \mathrm{RDA}$, and $\mathrm{DB}$ contributed equally to the interpretation of the data and writing of the manuscript. All authors read and approved the final manuscript.

\section{Ethics approval and consent to participate}

The protocol for METS was approved by the Institutional Review Board of Loyola University Chicago (IRB \#: LUC200038). Written, informed consent was obtained for each participant prior to any data collection.

\section{Competing interests}

The authors declare that they have no competing interests.

\section{Publisher's Note}

Springer Nature remains neutral with regard to jurisdictional claims in published maps and institutional affiliations.

Received: 23 June 2017 Accepted: 30 October 2017

Published online: 07 November 2017

\section{References}

1. Banerji MA, Buckley MC, Chaiken RL, Gordon D, Lebovitz HE, Kral JG. Liver fat, serum triglycerides and visceral adipose tissue in insulin-sensitive and insulin-resistant black men with NIDDM. Int J Obes Relat Metab Disord. 1995;19:846-50

2. Despres JP, Lemieux S, Lamarche B, et al. The insulin resistance-dyslipidemic syndrome: contribution of visceral obesity and therapeutic implications. Int J Obes Relat Metab Disord. 1995;19:S76-86.

3. Despres JP, Moorjani S, Lupien PJ, Tremblay A, Nadeau A, Bouchard C. Regional distribution of body fat, plasma lipoproteins, and cardiovascular disease. Arteriosclerosis. 1990;10:497-511.

4. Goodpaster BH, Krishnaswami S, Harris TB, et al. Obesity, regional fat distribution, and the metabolic syndrome in older men and women. Arch Intern Med. 2005;165:777-83. 
5. Hall JE, do Carmo JM, da Silva AA, Wang Z, Hall ME. Obesity-induced hypertension: interaction of neurohumoral and renal mechanisms. Circ Res. 2015;116:991-1006.

6. Nguyen-Duy TB, Nichaman MZ, Church TS, Blair SN, Ross R. Visceral fat and liver fat are independent predictors of metabolic risk factors in men. Am J Physiol Endocrinol Metab. 2003;284:E1065-71.

7. Pouliot MC, Despres JP, Nadeau A, et al. Visceral adiposity in men: associations with glucose tolerance, plasma insulin, and lipoprotein levels. Diabetes. 1992;41:826-34.

8. Vissers D, Hens W, Taeymans J, Baeyens JP, Poortmans J, Van Gaal L. The effect of exercise on visceral adipose tissue in overweight adults: a systematic review and meta-analysis. PLoS One. 2013;8(2):e56415.

9. Verheggen RJHM, Maessen MFH, Green DJ, Hermus ARMM, Hopman MTE, Thijssen DHT. A systemic review and meta-analysis on the effects of exercise training versus hypocaloric diet: distinct effects on body weight and visceral adipose tissue. Obes Rev. 2016;17:664-90.

10. Kuk JL, Katazmaryzk PT, Nichmann MZ, Church TS, Blair SN, Ross R. Visceral fat is an independent predictor of all-cause mortality in men. Obesity. 2006;14:336-41.

11. Pischon $\mathrm{T}$, Boeing $H$, Hoffman $\mathrm{K}$, et al. General and abdominal adiposity and risk of death in Europe. New Eng J Med. 2008;359:2105-20.

12. Alexopoulos N, Katritsis D, Raggi P. Visceral adipose tissue as a source of inflammation and promoter of atherosclerosis. Atherosclerosis. 2014;233:104-12.

13. DeMarco VG, Aroor AR, Sowers JR. The pathophysiology of hypertension in patients with obesity. Nat Rev Endocrinol. 2014;10:364-76.

14. Item F, Konrad D. Visceral fat and metabolic inflammation: the portal theory revisited. Obes Rev. 2012;13:30-9.

15. Strissel KJ, Denis GV, Nikolajczyk BS. Immune regulators of inflammation in obesity-associated type 2 diabetes and coronary artery disease. Curr Opin Endocrinol Diabetes Obes. 2014;21:330-8.

16. Colpani V, Oppermann K, Spritzer PM. Association between habitual physical activity and lower cardiovascular risk in premenopausal, perimenopausal, and postmenopausal women: a population-based study. Menopause. 2013;20:525-31.

17. Ekelund U, Franks PW, Sharp S, Brage S, Wareham NJ. Increase in physical activity energy expenditure is associated with reduced metabolic risk independent of change in fatness and fitness. Diabetes Care. 2007;30:2101-6.

18. Goedecke JH, Micklesfield LK. The effect of exercise on obesity, body fat distribution and risk for type 2 diabetes. Med Sport Sci. 2014;60:82-93.

19. Juonala M, Vikari JS, Raitakari OT. Main findings from the prospective cardiovascular risk in young Finns study. Curr Opin Lipidol. 2013;24:57-64.

20. Haskell WL, Lee IM, Pate RR, et al. Physical activity and public health: updated recommendations for adults from the American College of Sports Medicine and the American Heart Association. Circulation. 2007;116:1081-93.

21. U.S. Department of Health and Human Services. 2008 Physical Activity Guidelines for Americans. Washington, DC: U.S. Department of Health and Human Services; 2008. p. 21-4.

22. Healy GN, Wijndaele K, Dunstan DW, et al. Objectively measured sedentary time, physical activity, and metabolic risk: the Australian diabetes, obesity and lifestyle study (AusDiab). Diabetes Care. 2008;31:369-71.

23. Troiano RP, Berrigan D, Dodd KW, Masse LC, Tilert T, McDowell M. Physical activity in the United States measured by accelerometer. Med Sci Sport Exerc. 2008;40:181-8.

24. Philipsen $\mathrm{A}$, Hansen $\mathrm{AL}$, Jorgensen $\mathrm{ME}$, et al. Associations between objectively measured physical activity and abdominal fat distribution. Med Sci Sports Exerc. 2015:47:983-9.

25. Murabito JM, Pedley A, Massaro JM, et al. Moderate-to-vigorous physical activity with accelerometry is associated with visceral adipose tissue in adults. J Am Heart Assoc. 2015:4:e001379.

26. Zhang Q, Wang Y, Huang ES. Changes in racial/ethnic disparities in the prevalence of type 2 diabetes by obesity levels among US adults. Ethn Health. 2009;14:439-57.

27. Luke A, Bovet P, Forrester TE, et al. Protocol for the modeling the epidemiologic transition study: a longitudinal observational study of energy balance and change in body weight, diabetes and cardiovascular disease risk. BMC Public Health. 2011;11:927.

28. Luke A, Bovet P, Plange-Rhule J, et al. A mixed ecologic-cohort comparison of physical activity \& weight among young adults from five populations of African origin. BMC Public Health. 2014;14:397.

29. Puyau MR, Adolph AL, Vohra FA, Zakeri I, Butte NF. Prediction of activity energy expenditure using accelerometers in children. Med Sci Sports Exerc. 2004;36:1625-31.
30. Wong SL, Colley R, Connor Gorber S, Tremblay M. Actical accelerometer sedentary activity thresholds for adults. J Phys Act Health. 2011:8:587-91.

31. Kelly TL, Wilson KE, Ruth CR. Estimating visceral fat by dual-energy Xray absorptiometry. US patent application number US2010-0234719 (Hologic, Inc., 2010).

32. Kelly TL, Wilson KE, Ruth CR. Visceral fat measurement. US patent application number US2011-0235881 (Hologic, Inc., 2011).

33. Oh J-Y, Sung Y-A, Lee HJ. The visceral adiposity index as a predictor of insulin resistance in young women with polycystic ovary syndrome. Obesity. 2013;21:1690-4.

34. Micklesfield LK, Goedecke JH, Punyanitya M, Wilson KE, Kelly TL. Dual-energy $x$-ray performs as well as clinical computed tomography for the measurement of visceral fat. Obesity. 2012;20:1109-14.

35. Blake GM, Naeem M, Boutros M. Comparison of effective dose to children and adults from dual X-ray absorptiometry examinations. Bone. 2006;38:935-42.

36. Smith HA, Storti KL, Arena VC, et al. Associations between accelerometerderived physical activity and regional adiposity in young men and women. Obesity. 2013;21:1299-305.

37. Duscha BD, Slentz CA, Johnson $J$, et al. Effects of exercise training amount and intensity on peak oxygen consumption in middle-age men and women at risk for cardiovascular disease. Chest. 2005:128:2788-93.

38. Ross R, Dagnone D, Jones PJ, et al. Reduction in obesity and related comorbid conditions after diet-induced weight loss or exercise-induced weight loss in men. A randomized, controlled trial. Ann Intern Med. 2000; 133:92-103.

39. Ekelund U, Ward HA, Norat T, Luan J, May AM, Weiderpass E, Sharp SJ, Overvad K, Østergaard JN, Tjønneland A, Johnsen NF, Mesrine S, Fournier A, Fagherazzi G, Trichopoulou A, Lagiou P, Trichopoulos D, Li K, Kaaks R, Ferrari P, Licaj I, Jenab M, Bergmann M, Boeing H, Palli D, Sieri S, Panico S, Tumino $R$, Vineis P, Peeters PH, Monnikhof E, Bueno-de-Mesquita HB, Quirós JR, Aguda A, Sánchez MJ, Huerta JM, Ardanaz E, Arriola L, Hedblad B, Wifält SM, Johansson M, Key TJ, Travis RC, Khaw KT, Brage S, Wareham NJ, Riboli E. Physical activity and all-cause mortality across levels of overall and abdominal adiposity in European men and women: the European Prospective Investigation into Cancer and Nutrition Study (EPIC). Am J Clin Nutr. 2015;101:613-21.

\section{Submit your next manuscript to BioMed Central and we will help you at every step:}

- We accept pre-submission inquiries

- Our selector tool helps you to find the most relevant journal

- We provide round the clock customer support

- Convenient online submission

- Thorough peer review

- Inclusion in PubMed and all major indexing services

- Maximum visibility for your research

Submit your manuscript at www.biomedcentral.com/submit
Biomed Central 